\title{
Article \\ COVID-19 in Solid Organ Transplant Recipient: Exploring Cumulative Incidence, Seroprevalence and Risk Factors for Disease Severity
}

\author{
Rossana Caldara ${ }^{1,+}$, Paola Maffi ${ }^{1,2,+}$, Sabrina Costa ${ }^{3}$, Elena Bazzigaluppi ${ }^{3}$, Cristina Brigatti ${ }^{3}$, Vito Lampasona ${ }^{3}$, \\ Paola Magistretti ${ }^{3}$, Fabio Manenti ${ }^{3}$, Ilaria Marzinotto ${ }^{3}{ }^{D}$, Silvia Pellegrini ${ }^{3}\left(\mathbb{D}\right.$, Marina Scavini $^{3}$, \\ Antonio Secchi ${ }^{1,2, \ddagger}$ and Lorenzo Piemonti $2,3, *, \ddagger$ iD \\ check for \\ updates \\ 1 Clinical Transplant Unit, IRCCS Ospedale San Raffaele, 20132 Milan, Italy; caldara.rossana@hsr.it (R.C.); \\ maffi.paola@hsr.it (P.M.); secchi.antonio@hsr.it (A.S.) \\ 2 Faculty of Medicine and Surgery, Vita-Salute San Raffaele University, 20132 Milan, Italy \\ 3 Diabetes Research Institute, IRCCS Ospedale San Raffaele, 20132 Milan, Italy; costa.sabrina@hsr.it (S.C.); \\ bazzigaluppi.elena@hsr.it (E.B.); brigatti.cristina@hsr.it (C.B.); lampasona.vito@hsr.it (V.L.); \\ magistretti.paola@hsr.it (P.M.); manenti.fabio@hsr.it (F.M.); marzinotto.ilaria@hsr.it (I.M.); \\ pellegrini.silvia@hsr.it (S.P.); scavini.marina@hsr.it (M.S.) \\ * Correspondence: piemonti.lorenzo@hsr.it; Tel.: +39-02-26432706; Fax: 39-02-26432871 \\ + These authors contributed equally to this work as first authors. \\ $\ddagger$ These authors contributed equally to this work as last authors.
}

Citation: Caldara, R.; Maffi, P.; Costa, S.; Bazzigaluppi, E.; Brigatti, C.; Lampasona, V. Magistretti, P.; Manenti, F.; Marzinotto, I.; Pellegrini, S.; et al. COVID-19 in Solid Organ Transplant Recipient: Exploring Cumulative Incidence, Seroprevalence and Risk Factors for Disease Severity. Biology 2021, 10, 1349. https://doi.org/ 10.3390/biology10121349

Academic Editors: Ger Rijkers and Jean-Luc Murk

Received: 9 November 2021

Accepted: 13 December 2021

Published: 18 December 2021

Publisher's Note: MDPI stays neutral with regard to jurisdictional claims in published maps and institutional affiliations.

Copyright: (c) 2021 by the authors. Licensee MDPI, Basel, Switzerland. This article is an open access article distributed under the terms and conditions of the Creative Commons Attribution (CC BY) license (https:// creativecommons.org/licenses/by/ $4.0 /)$
Simple Summary: It is still uncertain whether recipients of solid organ transplant (SOT) are at increased risk of SARS-CoV-2 infection and/or poor outcomes due to COVID-19 in comparison to the general population. In this study, we report the cumulative incidence and outcomes of SARS-CoV-2 infection in a cohort of 291 SOT recipients. The COVID-19 cumulative incidence in SOT recipients resulted slightly higher compared to that of age-matched population during the study period. Moreover, the SARS-CoV-2 antibody frequency was around 2.6-fold higher than the incidence of cases who tested positive for SARS-CoV-2 RT-PCR, suggesting that the number of SOT recipients infected with SARS-CoV-2 is likely higher than described. In symptomatic recipients, kidney transplant was associated with a higher risk of developing moderate/critical disease, while common risk factors, including age and comorbidities, resulted less relevant for COVID-19 severity. Due to the high estimated crude mortality, symptomatic SOT recipients should be considered at high risk in case of SARS-CoV-2 infection.

Abstract: Background: Solid organ transplant (SOT) recipients may be at increased risk for severe disease and mortality from COVID-19 because of immunosuppression and prolonged end-stage organ disease. As a transplant center serving a diverse patient population, we report the cumulative incidence and outcomes of SARS-CoV-2 infection in our cohort of SOT recipients. Methods: We prospectively included in this observational study SOT recipients with a functioning kidney $(n=201)$, pancreas \pm kidney $(n=66)$ or islet transplant $(n=24)$, attending outpatient regular follow-up at the San Raffaele Hospital from February 2020 to April 2021. Antibodies to SARS-CoV-2 were tested in all patients by a luciferase immunoprecipitation system assay. Results: Of the 291 SOT recipients, $30(10.3 \%)$ tested positive for SARS-CoV-2 during the study period and prevalence was not different among different transplants. The SARS-CoV-2 antibody frequency was around 2.6-fold higher than the incidence of cases who tested positive for SARS-CoV-2 RT-PCR. As for the WHO COVID-19 severity classification, 19 (63.3\%) SOT recipients were mild, nine (30\%) were moderate, and two were critical and died yielding a crude mortality rate in our patient population of $6.7 \%$. Kidney transplant (OR $12.9(1.1-150) p=0.041)$ was associated with an increased risk for moderate/critical disease, while statin therapy (OR $0.116(0.015-0.926) p=0.042)$ and pancreas/islet transplant (OR $0.077(0.007-0.906)$ $p=0.041)$ were protective. Conclusions: The incidence of SARS-CoV-2 infection in SOT recipients may be higher than previously described. Due to the relative high crude mortality, symptomatic SOT recipients must be considered at high risk in case of SARS-CoV-2 infection. 


\section{Introduction}

On 21 February 2020 the first diagnosed case of COVID-19 was confirmed in Lombardy, a region of Northern Italy. On 8 March 2020, the entire Lombardy region went into lockdown with the rest of the country, and it quickly became a hotspot on the wold map of the COVID-19 pandemic. On 3 June 2020, free movement within the entire national territory was restored, de facto signaling the end of the SARS-CoV-2 associated disease first wave. Starting in July 2020, Italy witnessed a new progressive rise in COVID-19 cases, resulting in a second wave in November 2020 and a third wave in March 2021, as a result of the spreading of the Delta variant of SARS-CoV-2. Reports from the Italian National Institute of Health (Istituto Superiore di Sanità-ISS) document that most patients with COVID-19 show no or mild symptoms (52\% asymptomatic, $17 \%$ pauci-symptomatic and $21 \%$ with mild symptoms). Ten percent of the patients suffer disease at the severe end of the spectrum, with $3 \%$ progressing to critical disease with an overall case fatality rate of approximately $3 \%$ (reaching $>20 \%$ in individuals $>80$ year old) [1,2]. A more severe SARS-CoV-2 infection has been documented in patients with older age and with coexisting premorbid conditions, such as hypertension, morbid obesity, chronic kidney disease and diabetes [3]. Solid organ transplantation (SOT) recipients may be at increased risk for severe disease and mortality from COVID-19 disease due to immunosuppression and prolonged end-stage organ disease [4]. While some studies have suggested higher morbidity in SOT recipients [5-15], others did not confirm this evidence [15-20]. We report here the incidence and outcomes of SARS-CoV-2 infection in a cohort of 291 patients with kidney, pancreas or islet transplant, all of whom received regular follow-ups at the IRCCS Ospedale San Raffaele between February 2020 and April 2021.

\section{Methods}

\subsection{Study}

Recipients aged $\geq 18$ years with a functioning kidney, pancreas or islet transplant, attending the outpatient follow-up clinic at the IRCCS Ospedale San Raffaele between February 2020 and April 2021 were invited to enter this study. The study was completed in April 2021, as from this time on the transplanted subjects began to be vaccinated for SARS CoV-2. None of the included subjects had been vaccinated at the latest available follow-up. Three hundred and two out of $338(89.3 \%)$ accepted to participate and 291 out of $302(96.3 \%)$ completed the study. Most of the patients lived in Northern Italy $(n=245,84.2 \%)$, followed by those in Southern $(n=29,10 \%)$ and Central Italy $(n=17,5.8 \%)$. The study was approved by the Ethics Committee of the Ospedale San Raffaele and all patients signed a written informed consent prior to any study procedure. At the time of the first clinic access during their post-transplant follow up, a comprehensive medical assessment with detailed history and physical examination was performed in all patients. Data on all clinical characteristics, including clinical and pharmacological history, lifestyle factors, comorbidity and body measurements were collected in a structured data collection system. In particular, data on specific symptoms potentially correlated with COVID-19 were obtained asking participants to fill out a questionnaire. A luciferase immunoprecipitation system (LIPS) assay was used to test specific antibodies to different SARS-CoV-2 antigens, as previously described [21]. We tested for IgG antibodies to the virus' Receptor Binding Domain (RBD) and for IgG antibodies to a second antigenic region, the $\mathrm{S} 2$ domain of spike protein to apply a highly specific and sensitive strategy to monitor the SARS-CoV-2 humoral response [22]. After the first study visit, all SOT recipients were recommended to contact the transplant center in case of symptoms compatible with COVID-19 (passive monitoring) and provided periodic information on SARS-CoV-2 infection diagnosis by responding to a phone questionnaire (active monitoring). In case of hospitalization during the study period, clinical data 
were abstracted from the electronic medical record system of each hospital at which the patient presented. Each case of COVID-19 was defined by a positive reverse transcriptasepolymerase chain reaction (RT-PCR) result for SARS-CoV-2. All COVID-19 RT-PCR positive patients were classified for disease severity according to the WHO severity classification at diagnosis [23]. All COVID-19 RT-PCR positive patients were managed following the consensus promoted by the Italian Society of Organ Transplantation, the Italian Society of Nephrology, the Italian Society of Anesthesia and Intensive Care, and the Italian Group on Antimicrobial Stewardship [24].

\subsection{Statistical Analysis}

Median with inter-quartile range (IQR) was used to present continuous variables, and values were compared using the Mann-Whitney or Kruskal-Wallis test. Categorical variables are reported as frequency or percent and were compared using the Chi-square or Fischer's exact test, as appropriate. COVID-19 free survival was estimated according to Kaplan-Meier. The time-to-event was calculated from the date of the first locally diagnosed case of COVID-19 in Italy (21 February 2020) to the date of the positive RT-PCR result for SARS-CoV-2 or of the last follow-up visit, whichever occurred first. We studied the association between patient characteristics with time positive RT-PCR result for SARS-CoV2 using univariate Cox proportional hazards models. Effect estimates, adjusted for age and sex, were reported as hazard ratios (HRs) with the corresponding 95\% CI, estimated using the Wald approximation. Associations between baseline variables and COVID-19 severity was assessed by logistic regression. The effect estimates adjusted for age and sex were reported as odd ratios (ORs). Two-tailed $p$ values are reported, with $p$ value $<0.05$ indicating statistical significance. All confidence intervals are two-sided and not adjusted for multiple testing. Statistical analysis was performed with SPSS 24 (SPSS Inc./IBM) and GraphPad Prism version 5.04.

\section{Results}

We enrolled 290 patients with a kidney $(K, n=201)$, a pancreas (pancreas alone, $\mathrm{PA}=10$; pancreas-kidney, $\mathrm{PK}=56$ ) or islet transplant alone (ITA, $n=24)$ from the cohort of SOT recipients attending a regular follow-up at the IRCCS Ospedale San Raffaele. The baseline characteristics of the entire cohort are summarized in Table 1 . The overall median age of the cohort was $56(47-65)$ years, and 179 were male (61.5\%). Of the 291 SOT recipients, $30(10.3 \%)$ tested positive for SARS-CoV-2 RT-PCR during the study period (21 February 2020 to 24 April 2021, Figure 1a) and their characteristics are reported in Table 2. Most of the cases of positivity occurred during the second and third pandemic waves, while during the first wave the SOT recipients were mainly spared. COVID-19 prevalence was not different among different transplants: 20 out 201 for kidney (10\%), 7 out $66(10.7 \%)$ for pancreas \pm kidney and 3 out $24(12.5 \%)$ for islet (Figure 1a). The region of residence of SOT recipients did not influence COVID-19 prevalence: $10.6 \%$ of patients were form Northern Italy, $10.3 \%$ from Southern Italy and $5.9 \%$ from Central Italy $(p=0.825)$. There was no statistically significant association between the positivity for SARS-CoV-2 and age, body mass index, time since transplantation, type of transplant, comorbidities and ongoing therapies (Figure $1 \mathrm{~b}$ ) or immunosuppression intensity (triple vs. double/single regimen: HR $1.17(0.56-2.42) . p=0.68)$. All positive SOT recipients were classified for disease severity following the WHO severity classification at diagnosis: $19(63.3 \%)$ were mild, $9(30 \%)$ were moderate, and 2 were critical and died (Figure 2a) with a crude mortality rate of $6.7 \%$. No patient lost organ function during the SARS-CoV-2 infection. The most common symptoms at presentation were systemic (53.3\%: fever, fatigue/malaise, myalgia/arthralgia) and respiratory ( $40 \%$ : cough, dyspnea, sore throat, chest pain). Gastrointestinal symptoms were less prevalent (13.3\%: diarrhea, vomiting/plasma, abdominal) as well as other symptoms (26.7\%: headache, conjunctivitis, hypo/anosmia, hypo/dysgeusia, skin rash). Kidney transplant (OR $12.9(1.1-150) p=0.041)$ and anti-hypertensive therapy other than ACE system blockers, beta blockers and calcium channel antagonists (OR 7.07 (1.18-42.3) 
$p=0.032)$ were associated with an increased risk to develop moderate/critical disease. Statin therapy (OR $0.116(0.015-0.926) p=0.042)$ and pancreas/islet transplant (OR 0.077 $(0.007-0.906) p=0.041)$ were protective against moderate/critical disease (Figure $2 \mathrm{~b}$ ). Immunosuppression intensity was not significantly associated with an increased risk to develop moderate/critical disease (triple vs. double/single regimen: OR 2.25 (0.36-13.9); $p=0.38$ ). To estimate the prevalence of asymptomatic disease in our cohort, we analyzed the antibody response of the IgG class to the SARS-CoV-2 Spike protein (both RBD and S2) in all 291 patients at the time of their first study visit (median time after the first case in Italy: 242 days, IQR 201-292). Specific antibody response (positivity for both RBD and S2) was present in 16 out 291 patients (5.5\%). Of these, 6 participants were symptomatic and tested positive for SARS-CoV-2 RT-PCR before the study sampling (123 days (IQR 39-220) before), while no history of symptoms and/or positivity for SARS-CoV-2 RT-PCR were reported in the remaining 10 participants (62.5\%) (Figure 2c). As expected, all 24 participants who developed symptomatic disease and tested positive for SARS-CoV-2 RT-PCR after the study sampling (96 days (IQR 42-166) after) were negative for the antibody response against the virus.

Table 1. Baseline characteristics of the entire transplant cohort, and of patients with different type of transplant.

\begin{tabular}{|c|c|c|c|c|c|}
\hline Items & All & Islet & Pancreas \pm Kidney & Kidney & $p$ \\
\hline $\mathrm{N}$ & 291 & 24 & 66 & 201 & \\
\hline Age in years, median (IQR) & $56(47-65)$ & $51(36-60)$ & $54(47-59)$ & $57(49-66)$ & 0.001 \\
\hline Sex $M / F$ & $179 / 112$ & $11 / 13$ & $41 / 25$ & $127 / 74$ & 0.264 \\
\hline Race Caucasian (N (\%)) & $284(97.6)$ & $24(100)$ & $64(97)$ & $196(97.5)$ & 0.631 \\
\hline Body mass index $\left(\mathrm{kg} / \mathrm{m}^{2}\right)$ & $24.2(21.8-26.6)$ & $22.2(17.8-23.5)$ & $23.2(20-26.7)$ & $25(22.5-27)$ & $<0.001$ \\
\hline Months since transplant, median (IQR) & $53.4(17-121)$ & $79(34-131)$ & $75(24-160)$ & $48(12-106)$ & 0.005 \\
\hline \multicolumn{6}{|l|}{ Comorbidities $(\mathrm{N}(\%))$} \\
\hline Hypertension & $227(78)$ & $11(45.8)$ & $42(63.6)$ & $174(86.6)$ & $<0.001$ \\
\hline Coronary artery disease & $35(12)$ & $2(8.3)$ & $11(16.7)$ & $22(10.9)$ & 0.392 \\
\hline Chronic kidney disease & $156(53.6)$ & $2(8.3)$ & $29(43.9)$ & $125(62.2)$ & $<0.001$ \\
\hline Diabetes & $140(48.1)$ & $24(100)$ & $66(100)$ & $50(24.9)$ & $<0.001$ \\
\hline Peripheral vasculopathy & $30(10.3)$ & $1(4.2)$ & $7(10.6)$ & $22(10.9)$ & 0.585 \\
\hline Chronic obstructive pulmonary disease & $1(0.3)$ & $0(0)$ & $0(0)$ & $1(0.5)$ & 0.799 \\
\hline Neoplasia active & $27(9.3)$ & $1(4.2)$ & $3(4.5)$ & $23(11.4)$ & 0.164 \\
\hline Dyslipidemia & $35(12)$ & $2(8.3)$ & $5(7.6)$ & $28(13.9)$ & 0.327 \\
\hline Neuro degenerative disease & $2(0.7)$ & $1(4.2)$ & $1(1.5)$ & $0(0)$ & 0.043 \\
\hline \multicolumn{6}{|l|}{ Baseline therapy } \\
\hline Calcineurin inhibitor (CNI) & $270(92.8)$ & $21(87.5)$ & $64(97)$ & $185(92)$ & 0.235 \\
\hline $\begin{array}{l}\text { Mammalian target of rapamycin } \\
\text { inhibitors (mTORi) }\end{array}$ & 58 (19.9) & $9(37.5)$ & $3(4.5)$ & $46(22.9)$ & $<0.001$ \\
\hline Steroids & 133 (45.7) & $1(4.2)$ & $31(47)$ & $101(50.2)$ & $<0.001$ \\
\hline Mycophenolate mofetil & $218(74.9)$ & $11(45.8)$ & $59(89.4)$ & $148(73.6)$ & $<0.001$ \\
\hline Azathioprine & $18(6.2)$ & $6(25)$ & $4(6.1)$ & $8(4)$ & $<0.001$ \\
\hline \multicolumn{6}{|l|}{ "Intensity" of immunosuppression } \\
\hline - Triple regimen & $122(41.9)$ & $1(4.2)$ & $30(45.5)$ & $91(45.3)$ & \\
\hline CNI+antimetabolite+steroid & $102(83.6)$ & $0(0)$ & $28(93.3)$ & $74(81.3)$ & \\
\hline $\mathrm{CNI}+\mathrm{mTORi}+$ steroid & $14(11.5)$ & $0(0)$ & $1(3.3)$ & $13(14.3)$ & \\
\hline mTORi+antimetabolite+steroid & $5(4.1)$ & $0(0)$ & $1(3.3)$ & $4(4.4)$ & \\
\hline mTORi+CNI+antimetabolite & $1(0.8)$ & $1(100)$ & $0(0)$ & $0(0)$ & \\
\hline - Double regimen & $162(55.7)$ & $22(91.7)$ & $36(54.5)$ & $104(51.7)$ & \\
\hline CNI+antimetabolite & $118(72.8)$ & $13(59.1)$ & $34(94.4)$ & $71(68.3)$ & \\
\hline $\mathrm{CNI}+\mathrm{mTORi}$ & $28(17.3)$ & $6(27.3)$ & $0(0)$ & $22(21.2)$ & \\
\hline CNI+steroid & $5(3.1)$ & $1(4.5)$ & $1(2.8)$ & $3(2.9)$ & \\
\hline mTORi+steroid & $5(3.1)$ & $0(0)$ & $0(0)$ & $5(4.8)$ & \\
\hline 0 mTORi+antimetabolite & $4(2.5)$ & $2(9.1)$ & $1(2.8)$ & $1(1)$ & \\
\hline antimetabolite+steroid & $2(1.2)$ & $0(0)$ & $0(0)$ & $2(1.9)$ & \\
\hline
\end{tabular}


Table 1. Cont.

\begin{tabular}{|c|c|c|c|c|c|}
\hline Items & All & Islet & Pancreas \pm Kidney & Kidney & $p$ \\
\hline - Single regimen & $7(2.4)$ & $1(4.2)$ & $0(0)$ & $6(3)$ & \\
\hline O Antimetabolite & $4(57.1)$ & $1(100)$ & $0(0)$ & $3(50)$ & \\
\hline - CNI & $2(28.6)$ & $0(0)$ & $0(0)$ & $2(33.3)$ & \\
\hline mTORi & $1(14.3)$ & $0(0)$ & $0(0)$ & $1(16.7)$ & \\
\hline Ace inhibitors & $51(17.5)$ & $6(25)$ & $7(10.6)$ & $38(18.9)$ & 0.185 \\
\hline $\begin{array}{l}\text { Angiotensin II receptor type } 1 \\
\text { antagonists }\end{array}$ & $26(8.9)$ & $1(4.2)$ & $5(7.6)$ & $20(10)$ & 0.584 \\
\hline Beta blockers & $157(54)$ & $6(25)$ & $33(50)$ & $118(58.7)$ & 0.006 \\
\hline Calcium channel antagonists & $125(43)$ & $3(12.5)$ & $26(39.4)$ & $96(47.8)$ & 0.003 \\
\hline Other anti-hypertensive & $106(36.4)$ & $2(8.3)$ & $20(30.3)$ & $84(41.8)$ & 0.003 \\
\hline Aspirin & $185(63.6)$ & $6(25)$ & $45(68.2)$ & $134(66.7)$ & $<0.001$ \\
\hline Direct oral anticoagulant & $44(15.1)$ & $4(16.7)$ & $12(18.2)$ & $28(13.9)$ & 0.688 \\
\hline Statin & $134(46)$ & $7(29.2)$ & $27(40.9)$ & $100(49.8)$ & 0.102 \\
\hline Other hypolipidemic agents & $61(21)$ & $2(8.3)$ & $8(12.1)$ & $51(25.4)$ & 0.02 \\
\hline Insulin & $69(23.7)$ & $18(75)$ & $19(28.8)$ & $32(15.9)$ & $<0.001$ \\
\hline Hypoglycemic agent & $19(6.5)$ & $0(0)$ & $5(7.6)$ & $14(7)$ & 0.395 \\
\hline
\end{tabular}

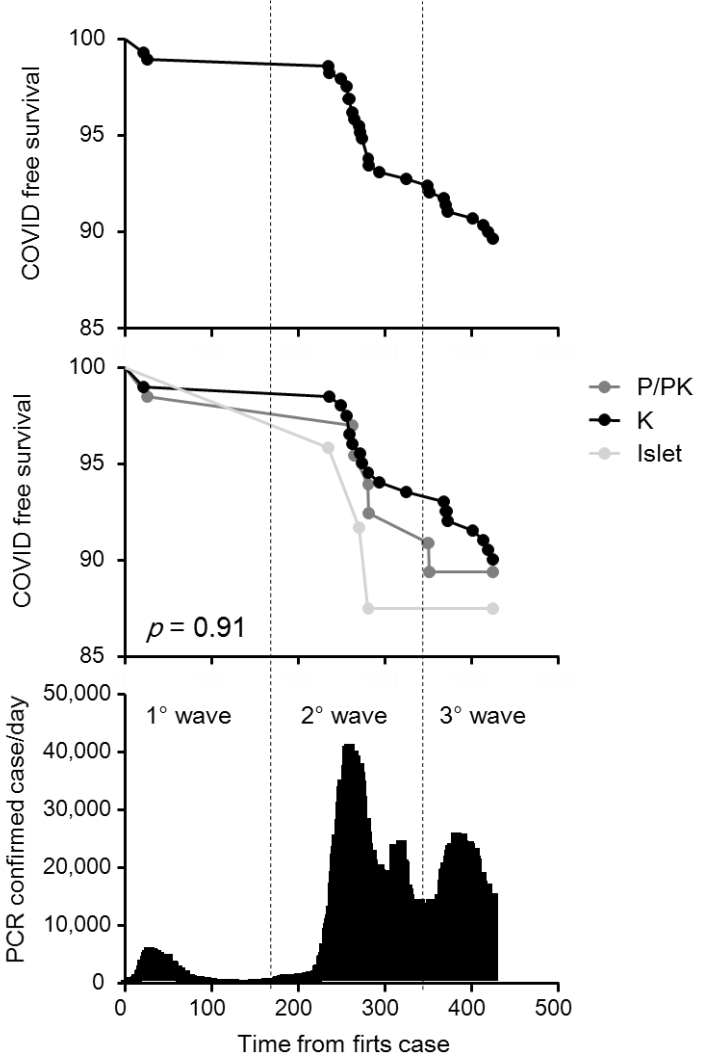

b

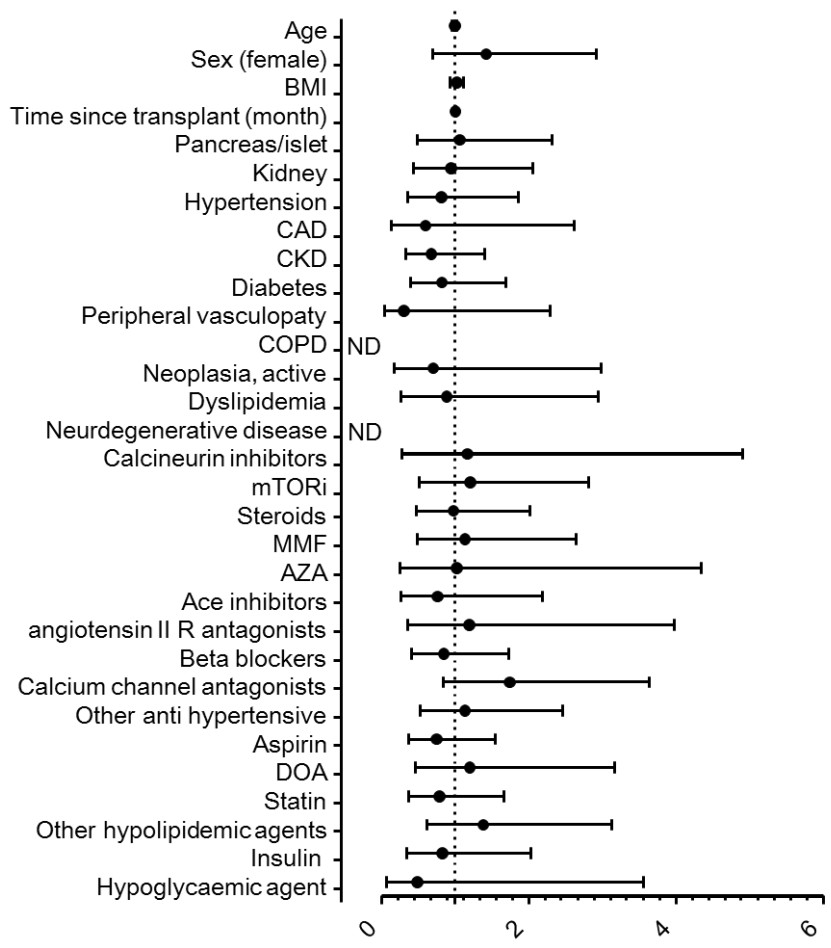

Hazard ratio for symptomatic PCR+ SARS-CoV-2 infection

Figure 1. COVID-19 RT-PCR positive subjects in solid organ transplantation (SOT) recipient. Kaplan-Meier COVID-free survival estimates for SOT recipients are in (panel a). Survival rate was estimated for all 291 SOT recipients (top panel) or according to the transplant type (kidney $(K, \mathrm{n}=201)$, pancreas (pancreas alone, $\mathrm{PA}=10$; pancreas-kidney, $\mathrm{PK}=56$ ), islet transplant alone (ITA: 24)) (middle panel) in relationship with the development of the COVID-19 pandemic in Italy (bottom panel). The log-rank test was used to test differences in the estimated survival rates among transplant types. The forest plot (panel b) shows the hazard ratios (HR) for positive RT-PCR for each factor tested. The univariate Cox regression analysis was adjusted for sex and age. Dots represent the HR, lines represent 95\% confidence interval (CI). 
a

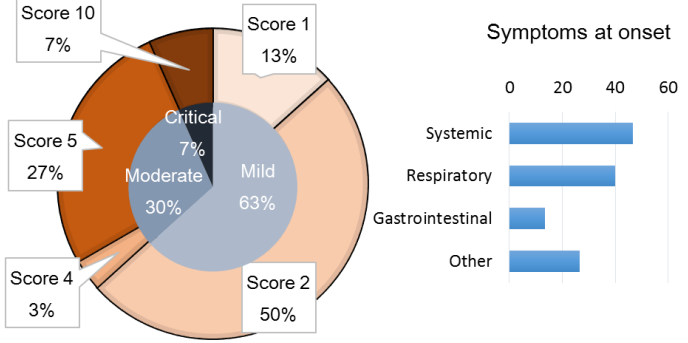

C

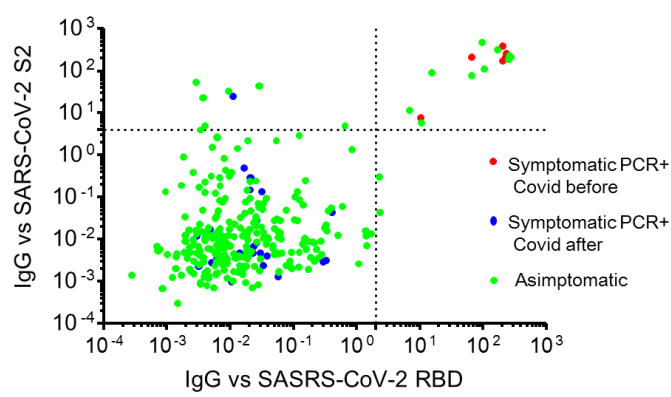

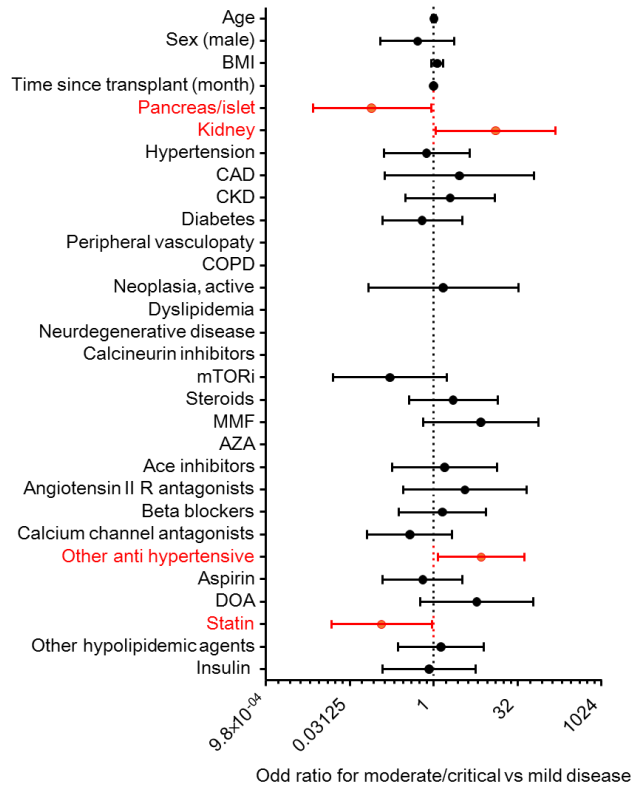

Figure 2. Clinical characteristic and SARS-CoV-2 antibody prevalence in COVID-19 RT-PCR positive subjects in solid organ transplantation (SOT) recipient. WHO severity classification [23] of COVID-19 RT-PCR positive subjects and prevalence of symptoms at diagnosis are in (panel a). Of the 291 SOT recipients, 30 (10.3\%) tested positive for SARS-CoV-2 RT-PCR during the study period (21 February 2020-24 April 2021). Presenting symptoms were classified as systemic (fever, fatigue/malaise, myalgia/arthralgia), respiratory (cough, dyspnea, sore throat, chest pain), gastrointestinal (diarrhea, vomiting/plasma, abdominal) and others (headache, conjunctivitis, hypo/anosmia, hypo/dysgeusia, skin rash). The forest plot (panel b) shows the odd ratios (OR) for moderate/critical disease for each factor tested. The univariate logistic regression analysis was adjusted for sex and age. Dots represent the HR, lines represent 95\% confidence interval (CI), and red dots indicate $p<0.05$. The dot plot (panel c) shows the value of IgG antibodies to the virus receptor binding domain (RBD) and the S2 domain of the spike protein. Patients were classified as asymptomatic (green), and symptomatic with a positive SARS-CoV-2 RT-PCR before the antibody test (red) and after the antibody test (blue). Dotted lines indicate the cut-off of antibody test positivity.

Table 2. Characteristics of SOT recipients that tested positive for SARS-CoV-2 RT-PCR during the study period.

\begin{tabular}{|c|c|c|c|}
\hline Items & SARS-CoV-2 RT-PCR Negative & SARS-Cov-2 RT-PCR Positive & $p$ \\
\hline $\mathrm{N}$ & 261 & 30 & \\
\hline Age in years, median (IQR) & $56(47-65)$ & $52(48-61)$ & 0.341 \\
\hline Sex M/F & $163 / 98$ & $16 / 14$ & 0.331 \\
\hline Race Caucasian (N (\%)) & $256(98.1)$ & $28(93.3)$ & 0.156 \\
\hline Body mass index $\left(\mathrm{kg} / \mathrm{m}^{2}\right)$ & $24.2(21.8-26.6)$ & $24(21.9-26.9)$ & 0.817 \\
\hline \multicolumn{4}{|l|}{ Type of transplant } \\
\hline - Kidney & $181(69.3)$ & $20(66.7)$ & \\
\hline - Pancreas \pm kidney & $59(22.6)$ & $7(23.3)$ & 0.924 \\
\hline - Islets & $21(8)$ & $3(10)$ & \\
\hline \multicolumn{4}{|l|}{ Comorbidities (N (\%)) } \\
\hline - Hypertension & $205(78.5)$ & $22(73.3)$ & 0.492 \\
\hline - Coronary artery disease & 33 (12.6) & $2(6.7)$ & 0.552 \\
\hline - Chronic kidney disease & $143(54.8)$ & $13(43.3)$ & 0.251 \\
\hline - Diabetes & $127(48.7)$ & $13(43.3)$ & 0.7 \\
\hline - Peripheral vasculopathy & 29 (11.1) & $1(3.3)$ & 0.337 \\
\hline - Chronic obstructive pulmonary disease & $1(0.4)$ & $0(0)$ & 1 \\
\hline - Neoplasia active & $25(9.6)$ & $2(6.7)$ & 1 \\
\hline - Dyslipidemia & 32 (12.3) & $3(10)$ & 1 \\
\hline - Neuro degenerative disease & $2(0.8)$ & $0(0)$ & 1 \\
\hline \multicolumn{4}{|l|}{ Baseline therapy } \\
\hline - Calcineurin inhibitor $(\mathrm{CNI})$ & $242(92.7)$ & $28(93.3)$ & 1 \\
\hline $\begin{array}{l}\text { - Mammalian target of rapamycin inhibitors } \\
\text { (mTORi) }\end{array}$ & $51(19.5)$ & $7(23.3)$ & 0.631 \\
\hline - Steroids & 119 (45.6) & $14(46.7)$ & 1 \\
\hline - Antimetabolites & $211(80.8)$ & $25(83.3)$ & 1 \\
\hline \multicolumn{4}{|l|}{ "Intensity" of immunosuppression } \\
\hline - Triple regimen & $108(41.4)$ & $14(46.7)$ & \\
\hline - Double regimen & $146(55.9)$ & $16(50.2)$ & 0.421 \\
\hline - Single regimen & $7(2.7)$ & $0(0)$ & \\
\hline
\end{tabular}




\section{Discussion}

The impact of SARS-CoV-2 infection in SOT recipients is an area of intense investigation. Here we present the results of a study in a cohort of SOT recipients undergoing regular follow-up at our institute. Our study generated several interesting findings. First, the COVID-19 cumulative incidence in SOT recipients (10.3\%) was slightly higher compared to that of the overall Italian population $(6.54 \%)$ or age-matched population (age 50-59: 7.4\%; age 60-69: 5.9\%) during the study period (21 February-24 April 2021; public data from ISS). The modest increase is also evident when compared with the cumulative incidence in Lombardy (7.89\% on 24 April 2021), one of the Italian regions most affected by the pandemic and in which $64 \%$ of the SOT recipients of our patient population resides. Our data are open to multiple interpretations. The higher cumulative COVID-19 incidence could be to the result of a higher susceptibility to the SARS-CoV-2 infection, to the propensity to refer to healthcare services even in the case of mild symptoms, or to a greater susceptibility to develop a symptomatic form. Second, at the time of the first study visit during post-transplant follow-up we found an overall SARS-CoV-2 antibody prevalence substantially higher than the cumulative incidence of diagnosed SARS-CoV-2 cases. The antibody frequency was approximately 2.6-fold higher than the incidence of SARS-CoV-2 RT-PCR positive SOT recipients, of whom more than half did not report symptoms. As previously reported [25], the specificity of the testing strategy can largely influence the estimated frequency of infection, potentially leading to greatly overestimated population prevalence. For this reason, we applied a two-stage approach, which provided a sensitive and specific approach for detecting SARS-CoV-2 antibodies [22]. Our antibody results underline that testing only patients with symptoms is not effective for identifying all infected SOT recipients. This suggests that the number of SOT recipients infected with SARS-CoV-2 is likely higher than described and that it is difficult to estimate the true impact of the infection in this specific population. Third, we found no association between cumulative incidence and characteristics such as sex, age, body mass index, time since transplantation, type of transplant, comorbidities and ongoing therapies. Fourth, the disease severity in SOT recipients was comparable to that reported in age-matched patients in the general population during the study period (mild disease 68\% (asymptomatic: $50 \%$ + paucisymptomatic $18 \%$ ), moderate $23 \%$, severe/critical $9 \%$; public data from ISS), but the estimated crude mortality was higher (age 50-59: 0.6\%; age 60-69: 2.7\%; public data from ISS). Interestingly, we found no association between disease severity and preexisting comorbidities including hypertension, diabetes, and obesity. Nevertheless, kidney transplant was identified as a risk factor predicting disease severity, while pancreas/islet transplant and statin therapy were protective factors. The fact that COVID-19 severity may be influenced by the transplant type was already suggested. In a recent metanalysis by Raja et al. [26], all-cause mortality in the subset of kidney transplant recipients was $22.0 \%$, vs. $11.8 \%$ among liver transplant recipients and $15.6 \%$ among heart transplant recipients. In part, this was explained by the high prevalence of acute kidney injury $(45.4 \%)$ and bacterial urinary tract infection in kidney transplant recipients [26]. Of particular interest, we have, for the first time, reported data about a cohort of islet transplant recipients, who showed a behavior similar to that of whole-organ recipients. Regarding statin therapy, it is a matter of discussion whether its use is associated with a reduced severity or mortality for COVID-19. Conflicting results were published and subgroup analyses suggested the existence of a different susceptibility to statin benefit during SARS-CoV-2 infection [27-29]. The suggestion that SOT recipients could benefit from statins is relevant in selecting the target population for future randomized clinical trials.

Our study has several limitations. First, although we tried to capture all cases of SARS-CoV-2 infection in our transplant cohort, it is possible that patients with very mild symptoms consistent with SARS-CoV-2 did not undergo RT-PCR testing. Second, the fact that we did not observe major differences in disease severity with increasing age or between patients with or without relevant comorbidities, could be due to the relatively small number of cases, narrow age range of recipients and low comorbid heterogeneity observed in our 
cohort. Third, although all efforts have been made to manage transplant recipients with SARS-CoV-2 infection as recommended by current guidelines, different treatments related to local standards and research protocols from other hospitals may have produced some heterogeneity across pandemic waves [30]. Fourth, the result that renal transplants are at higher risk for moderate-severe COVID-19 when compared to islet or pancreas (w/kidney) transplants is interesting, but the groups had distinct baseline characteristics. In fact, islet and pancreas ( $\mathrm{w} /$ kidney) recipients had lower BMI, longer interval from transplant, and lower incidence of hypertension and chronic kidney disease. Unfortunately, the number of events in our cohort are not sufficient to perform a multivariate analysis and, even if in our cohort these single variables did not result in a higher risk for moderate/critical disease, we cannot exclude their potential role in determining the result.

\title{
5. Conclusions
}

In conclusion, the incidence of SARS-CoV-2 infection in SOT recipients is likely higher than previously described, as suggested by the presence of viral antibodies, and indicates that estimating the true impact of the infection on clinical outcomes is challenging. In symptomatic recipients, kidney transplant was associated with a higher risk to develop moderate/critical disease, while common risk factors, including age and comorbidities, resulted less relevant for COVID-19 severity. Due to the high estimated crude mortality, symptomatic SOT recipients should be considered at high risk in case of SARS-CoV-2 infection. However, further studies are needed to identify predictive biomarkers and risk factors for disease outcome.

Author Contributions: L.P., R.C. and P.M. (Paola Maffi) contributed to the conception of the study, wrote the manuscript, researched data and contributed to the discussion. E.B., C.B., V.L., P.M. (Paola Magistretti), S.P., S.C., F.M. and I.M. contributed to the acquisition of samples, managed the bio-banking activities, and performed the antibodies assay. A.S. and M.S. contributed to the acquisition, analysis and interpretation of clinical data, and critically reviewed/edited the manuscript. All authors have read and agreed to the published version of the manuscript.

Funding: This work was funded by Program Project COVID-19 OSR-UniSR and Ministero della Salute (COVID-2020-12371617).

Institutional Review Board Statement: The study was conducted according to the guidelines of the Declaration of Helsinki, and approved by the Ethics Committee of Ospedale San Raffaele. Data and sample collection was made under the BIODRI project (approved 07/11/2019) and COVID-BioB project (approved 19 March 2020: NCT04318366). The samples and data analysis were approved under the project SIERO COVID (approved 15 July 2020: NCT04487197).

Informed Consent Statement: Informed consent was obtained from all subjects involved in the study.

Data Availability Statement: The data presented in this study are available on request from the corresponding author. The data are not publicly available due to privacy restrictions.

Acknowledgments: This study was supported by Program Project COVID-19 OSR-UniSR and Ministero della Salute (COVID-2020-12371617).

Conflicts of Interest: The authors have no conflict of interest to disclose in relation to the topic of this manuscript. The authors declare that there are no relationships or activities that might bias, or be perceived to bias, their work.

\author{
Abbreviations \\ K Kidney \\ IQR inter-quartile range \\ ITA Islet transplant alone \\ ISS Istituto Superiore di Sanità \\ LIPS luciferase immunoprecipitation system \\ OD Odd ratio \\ PA Pancreas alone
}




$\begin{array}{ll}\text { PK } & \text { Pancreas-kidney } \\ \text { RBD } & \text { Receptor binding domain } \\ \text { RT-PCR } & \text { reverse transcriptase-polymerase chain reaction } \\ \text { SARS-CoV-2 } & \text { severe acute respiratory syndrome coronavirus 2 } \\ \text { SOT } & \text { Solid organ transplantation }\end{array}$

\section{References}

1. Unim, B.; Palmieri, L.; Lo Noce, C.; Brusaferro, S.; Onder, G. Prevalence of COVID-19-related symptoms by age group. Aging Clin. Exp. Res. 2021, 33, 1145-1147. [CrossRef]

2. Palmieri, L.; Vanacore, N.; Donfrancesco, C.; Lo Noce, C.; Canevelli, M.; Punzo, O.; Raparelli, V.; Pezzotti, P.; Riccardo, F.; Bella, A. Clinical characteristics of hospitalized individuals dying with COVID-19 by age group in Italy. J. Gerontol. Ser. A 2020, 75, 1796-1800. [CrossRef] [PubMed]

3. Wingert, A.; Pillay, J.; Gates, M.; Guitard, S.; Rahman, S.; Beck, A.; Vandermeer, B.; Hartling, L. Risk factors for severity of COVID-19: A rapid review to inform vaccine prioritisation in Canada. BMJ Open 2021, 11, e044684. [CrossRef] [PubMed]

4. Azzi, Y.; Bartash, R.; Scalea, J.; Loarte-Campos, P. Akalin E COVID-19 and Solid Organ Transplantation: A Review Article. Transplantation 2021, 105, 37-55. [CrossRef] [PubMed]

5. Pereira, M.R.; Mohan, S.; Cohen, D.J.; Husain, S.A.; Dube, G.K.; Ratner, L.E.; Arcasoy, S.; Aversa, M.M.; Benvenuto, L.J.; Dadhania, D.M.; et al. COVID-19 in solid organ transplant recipients: Initial report from the US epicenter. Am. J. Transpl. 2020, 20, 1800-1808. [CrossRef] [PubMed]

6. Akalin, E.; Azzi, Y.; Bartash, R.; Seethamraju, H.; Parides, M.; Hemmige, V.; Ross, M.; Forest, S.; Goldstein, Y.D.; Ajaimy, M.; et al. Covid-19 and Kidney Transplantation. N. Engl. J. Med. 2020, 382, 2475-2477. [CrossRef] [PubMed]

7. Caillard, S.; Anglicheau, D.; Matignon, M.; Durrbach, A.; Greze, C.; Frimat, L.; Thaunat, O.; Legris, T.; Moal, V.; Westeel, P.F.; et al. An initial report from the French SOT COVID Registry suggests high mortality due to COVID-19 in recipients of kidney transplants. Kidney Int. 2020, 98, 1549-1558. [CrossRef] [PubMed]

8. Roberts, M.B.; Izzy, S.; Tahir, Z.; Al Jarrah, A.; Fishman, J.A.; El Khoury, J. COVID-19 in solid organ transplant recipients: Dynamics of disease progression and inflammatory markers in ICU and non-ICU admitted patients. Transpl. Infect. Dis. 2020, 22, e13407. [CrossRef] [PubMed]

9. Fishman, J.A. The Immunocompromised Transplant Recipient and SARS-CoV-2 Infection. J. Am. Soc. Nephrol. 2020, 31, 1147-1149. [CrossRef] [PubMed]

10. Fernandez-Ruiz, M.; Andres, A.; Loinaz, C.; Delgado, J.F.; Lopez-Medrano, F.; San Juan, R.; Gonzalez, E.; Polanco, N.; Folgueira, M.D.; Lalueza, A.; et al. COVID-19 in solid organ transplant recipients: A single-center case series from Spain. Am. J. Transpl. 2020, 20, 1849-1858. [CrossRef]

11. Crespo, M.; Perez-Saez, M.J.; Redondo-Pachon, D.; Llinas-Mallol, L.; Montero, M.M.; Villar-Garcia, J.; Arias-Cabrales, C.; Buxeda, A.; Burballa, C.; Vazquez, S.; et al. COVID-19 in elderly kidney transplant recipients. Am. J. Transpl. 2020, 20, $2883-2889$. [CrossRef] [PubMed]

12. Kates, O.S.; Haydel, B.M.; Florman, S.S.; Rana, M.M.; Chaudhry, Z.S.; Ramesh, M.S.; Safa, K.; Kotton, C.N.; Blumberg, E.A.; Besharatian, B.D.; et al. COVID-19 in solid organ transplant: A multi-center cohort study. Clin. Infect. Dis. 2020, 73, 4090-4099. [CrossRef] [PubMed]

13. Aversa, M.; Benvenuto, L.; Anderson, M.; Shah, L.; Robbins, H.; Pereira, M.; Scheffert, J.; Carroll, M.; Hum, J.; Nolan, M.; et al. COVID-19 in lung transplant recipients: A single center case series from New York City. Am. J. Transpl. 2020, 20, 3072-3080. [CrossRef] [PubMed]

14. Cravedi, P.; Mothi, S.S.; Azzi, Y.; Haverly, M.; Farouk, S.S.; Perez-Saez, M.J.; Redondo-Pachon, M.D.; Murphy, B.; Florman, S.; Cyrino, L.G.; et al. COVID-19 and kidney transplantation: Results from the TANGO International Transplant Consortium. Am. J. Transpl. 2020, 20, 3140-3148. [CrossRef] [PubMed]

15. Avery, R.K.; Chiang, T.P.; Marr, K.A.; Brennan, D.C.; Sait, A.S.; Garibaldi, B.T.; Shah, P.; Ostrander, D.; Steinke, S.M.; Permpalung, N.; et al. Inpatient COVID-19 outcomes in solid organ transplant recipients compared to non-solid organ transplant patients: A retrospective cohort. Am. J. Transpl. 2021, 21, 2498-2508. [CrossRef]

16. Bossini, N.; Alberici, F.; Delbarba, E.; Valerio, F.; Manenti, C.; Possenti, S.; Econimo, L.; Maffei, C.; Pola, A.; Terlizzi, V.; et al. Kidney transplant patients with SARS-CoV-2 infection: The Brescia Renal COVID task force experience. Am. J. Transpl. 2020, 20, 3019-3029. [CrossRef]

17. Fava, A.; Cucchiari, D.; Montero, N.; Toapanta, N.; Centellas, F.J.; Vila-Santandreu, A.; Coloma, A.; Meneghini, M.; Manonelles, A.; Sellares, J.; et al. Clinical characteristics and risk factors for severe COVID-19 in hospitalized kidney transplant recipients: A multicentric cohort study. Am. J. Transpl. 2020, 20, 3030-3041. [CrossRef] [PubMed]

18. Molnar, M.Z.; Bhalla, A.; Azhar, A.; Tsujita, M.; Talwar, M.; Balaraman, V.; Sodhi, A.; Kadaria, D.; Eason, J.D.; Hayek, S.S.; et al. Outcomes of critically ill solid organ transplant patients with COVID-19 in the United States. Am. J. Transpl. 2020, 20, 3061-3071. [CrossRef]

19. Avery, R.K. COVID-19 Therapeutics for Solid Organ Transplant Recipients; 6 Months Into the Pandemic: Where Are We Now? Transplantation 2021, 105, 56-60. [CrossRef] [PubMed] 
20. Hadi, Y.B.; Naqvi, S.F.Z.; Kupec, J.T.; Sofka, S.; Sarwari, A. Outcomes of COVID-19 in Solid Organ Transplant Recipients: A Propensity-matched Analysis of a Large Research Network. Transplantation 2021, 105, 1365-1371. [CrossRef]

21. Secchi, M.; Bazzigaluppi, E.; Brigatti, C.; Marzinotto, I.; Tresoldi, C.; Rovere-Querini, P.; Poli, A.; Castagna, A.; Scarlatti, G.; Zangrillo, A.; et al. COVID-19 survival associates with the immunoglobulin response to the SARS-CoV-2 spike receptor binding domain. J. Clin. Invest. 2020, 130, 6366-6378. [CrossRef]

22. Hippich, M.; Holthaus, L.; Assfalg, R.; Zapardiel-Gonzalo, J.; Kapfelsperger, H.; Heigermoser, M.; Haupt, F.; Ewald, D.A.; Welzhofer, T.C.; Marcus, B.A.; et al. A Public Health Antibody Screening Indicates a 6-Fold Higher SARS-CoV-2 Exposure Rate than Reported Cases in Children. Medicine 2021, 2, 149-163. [CrossRef]

23. Marshall, J.C.; Murthy, S.; Diaz, J.; Adhikari, N.K.; Angus, D.C.; Arabi, Y.M.; Baillie, K.; Bauer, M.; Berry, S.; Blackwood, B.; et al. A minimal common outcome measure set for COVID-19 clinical research. Lancet Infect. Dis. 2020, 20, 192-197. [CrossRef]

24. Vistoli, F.; Furian, L.; Maggiore, U.; Caldara, R.; Cantaluppi, V.; Ferraresso, M.; Zaza, G.; Cardillo, M.; Biancofiore, G.; Menichetti, F.; et al. COVID-19 and kidney transplantation: An Italian Survey and Consensus. J. Nephrol. 2020, 33, 667-680. [CrossRef]

25. Deeks, J.J.; Dinnes, J.; Takwoingi, Y.; Davenport, C.; Spijker, R.; Taylor-Phillips, S.; Adriano, A.; Beese, S.; Dretzke, J.; di Ruffano, L.F. Antibody tests for identification of current and past infection with SARS-CoV-2. Cochrane Database Syst. Rev. 2020. [CrossRef]

26. Raja, M.A.; Mendoza, M.A.; Villavicencio, A.; Anjan, S.; Reynolds, J.M.; Kittipibul, V.; Fernandez, A.; Guerra, G.; Camargo, J.F.; Simkins, J.; et al. COVID-19 in solid organ transplant recipients: A systematic review and meta-analysis of current literature. Transplant. Rev. 2021, 35, 100588. [CrossRef]

27. Wu, K.S.; Lin, P.C.; Chen, Y.S.; Pan, T.C.; Tang, P.L. The use of statins was associated with reduced COVID-19 mortality: A systematic review and meta-analysis. Ann. Med. 2021, 53, 874-884. [CrossRef] [PubMed]

28. Scheen, A.J. Statins and clinical outcomes with COVID-19: Meta-analyses of observational studies. Diabetes Metab. 2020, 47, 101220. [CrossRef] [PubMed]

29. Hariyanto, T.I.; Kurniawan, A. Statin and outcomes of coronavirus disease 2019 (COVID-19): A systematic review, meta-analysis, and meta-regression. Nutr. Metab. Cardiovasc. Dis. 2021, 31, 1662-1670. [CrossRef] [PubMed]

30. Coll, E.; Fernandez-Ruiz, M.; Padilla, M.; Moreso, F.; Hernandez-Vicente, A.; Yanez, I.; Molina, M.; Vazquez-Sanchez, T.; Crespo, M.; Facundo, C.; et al. COVID-19 in Solid Organ Transplant Recipients in Spain Throughout 2020: Catching the Wave? Transplantation 2021, 105, 2146. [CrossRef] 